\title{
The young stellar cluster associated with IRAS 09149-4743 $\star, \star \star$
}

\author{
R. Ortiz ${ }^{1}$, A. Roman-Lopes ${ }^{2}$, and Z. Abraham ${ }^{2}$ \\ 1 Escola de Artes, Ciências e Humanidades, USP, Av. Arlindo Bettio, 1000, 03828-000, São Paulo, SP, Brazil \\ e-mail: ortiz@astro.iag.usp.br \\ 2 Departamento de Astronomia, IAG/USP, Rua do Matão, 1226, Cidade Universitária, 05508-900, São Paulo, SP, Brazil
}

Received 10 November 2005 / Accepted 20 August 2006

\begin{abstract}
Context. We present the results of a near-infrared survey of the young stellar cluster associated with IRAS 09149-4743, a member of the Vela Molecular Ridge.

Aims. The main purpose of this survey is to study the cluster members and find the ionizing sources of the associated HII region. Methods. Infrared photometry of 62 stars located in the crowded parts of the cluster is given in the $J, H$, and $K$ bands. Colour-colour and colour-magnitude diagrams are drawn to obtain an estimate of their spectral type and reddening.

Results. Three main sources are identified as candidates to ionize the HII region: a star located at the centre of the cluster, inside the IRAS error ellipse; a second star, member of a small "subcluster" situated 1.1' to the southeast of the former; and a third object, showing very high colour indices, which indicate visual extinction over 20 mag. Dereddened $J$ magnitudes suggest that the former two stars probably are the main ionizing sources, whereas the latter is too reddened to allow a reliable estimate of its spectral type by photometric methods.
\end{abstract}

Key words. stars: formation - ISM: HII regions - Galaxy: open clusters and associations: individual: IRAS 09149-4743 infrared: stars

\section{Introduction}

Among the various molecular clouds where star formation is currently taking place, the Vela Molecular Ridge (VMR) is one of the best candidates for the detection of young stellar objects. Situated near the tangential direction of the Carina arm, it extends over 20 degrees in the sky, comprising four main complexes with active star formation, named $A$ to $D$ (Murphy \& May 1991). Observations of the CO $J=1-0$ line, carried out over the entire complex (May et al. 1988; Murphy \& May 1991), revealed numerous dense regions, with strong emission of CS lines (e.g. Zinchenko et al. 1995; Bronfman et al. 1996; Lapinov et al. 1998). This molecule is a well-known tracer of high-density molecular clouds because its excitation occurs only at densities greater than $10^{4}-10^{5} \mathrm{~cm}^{-3}$ (Snell et al. 1984). Several infrared surveys have been carried out towards the VMR which have shown that star formation is currently taking place over a wide range of masses (Massi et al. 1999, 2000, 2003). Their results show that more luminous $\left(L_{\mathrm{bol}}>10^{3} L_{\odot}\right)$, massive stars are being formed in clusters with a high density of stars.

IRAS 09149-4743, situated in cloud $A$ of VMR, is associated with an HII region about $5.2^{\prime}$ in size, detected in the continuum and recombination lines (Caswell \& Haynes 1987), which suggests the presence of massive stars. It is a well known fact that massive young stellar objects often appear related to methanol masers, especially to the transition at $6.67 \mathrm{GHz}$

^ Based on observations obtained at Pico dos Dias Observatory, operated by the National Laboratory for Astrophysics, LNA, Brazil.

$\star \star$ Table 2 is only available in electronic form at the CDS via anonymous ftp to cdsarc.u-strasbg.fr (130.79.128.5) or via http://cdsweb.u-strasbg.fr/cgi-bin/qcat?J/A+A/461/949
(Menten 1991). In fact, maser emission at this frequency was found near IRAS 09149-4743 by Caswell et al. (1995) and Walsh et al. (1997) which suggests the existence of an ultra-compact (UC) HII region, generally found near O or early B spectral type stars. At the same position, Dutra et al. (2003) list an infrared stellar cluster detected in the 2MASS survey, catalogued under \#36, measuring 1.2' in diameter.

Although the 2MASS survey (Skrutskie et al. 1997) constitutes an important tool to identify young star forming regions severely obscured by dust, its limited spatial resolution does not allow the separation of individual stars in crowded fields, as occurs in the cluster associated with IRAS 09149-4743. In this work we present the results of a more precise study of this young cluster in the near-infrared $J, H$, and $K$ bands. In Sect. 2 previous infrared, molecular and continnuum data are presented to gather a complete scenario of the region; our new infrared observations of the cluster are described in Sect. 3. The physical study of the cluster, including colour-colour and colour-magnitude diagrams are presented in Sect. 4.

\section{The molecular cloud associated with IRAS 09149-4743}

Rodgers et al. (1960) detected an $\mathrm{H} \alpha$ emission region (RCW 41) in the direction of IRAS 09149-4743, measuring $8^{\prime} \times 4^{\prime}$. Later, Brand et al. (1986), examining plates of the ESO/SRC survey, also catalogued a nebulous object of about $6^{\prime}$ in size, but only molecular observations reveal that this object is actually part of a molecular cloud where massive star formation commonly takes place. In Table 1 the main radio and infrared observations of the region near IRAS 09149-4743 available in the literature are 
Table 1. Main radio and infrared observations near IRAS 09149-4743 collected from the literature. The position offsets are relative to the IRAS source located at: $\alpha(1950.0)=9 \mathrm{~h} 14 \mathrm{~m} 57.9 \mathrm{~s}, \delta(1950.0)=-47^{\circ} 43^{\prime} 50^{\prime \prime}$.

\begin{tabular}{|c|c|c|c|c|c|c|c|c|}
\hline Transition & Frequency & $\operatorname{LSR}\left(\mathrm{km} \mathrm{s}^{-1}\right)$ & $\alpha$ offset (") & $\delta$ offset $\left({ }^{\prime \prime}\right)$ & $H P B W\left({ }^{\prime \prime}\right)$ & Intensity & Map & Reference \\
\hline $\mathrm{CO}(1-0)$ & $115.271 \mathrm{GHz}$ & 9.8 & 0 & 0 & 43 & $22.9 \mathrm{~K}$ & $\bar{N}$ & WB89 \\
\hline $\mathrm{CO}(1-0)$ & $115.271 \mathrm{GHz}$ & $5.9-9.8$ & - & - & $5.3 \times 10^{2}$ & - & $\mathrm{Y}$ & MM91 \\
\hline $\mathrm{CO}(1-0)$ & $115.271 \mathrm{GHz}$ & $7.0 / 10.0$ & -40 & +30 & 45 & $14 / 44 \mathrm{~K}$ & $\mathrm{~N}$ & ZMT95 \\
\hline $\mathrm{CO}(1-0)$ & $115.271 \mathrm{GHz}$ & 8.82 & -40 & +30 & 45 & $43.7 \mathrm{~K}$ & $\mathrm{~N}$ & Lap98 \\
\hline $\mathrm{C}^{18} \mathrm{O}(1-0)$ & $109.782 \mathrm{GHz}$ & 9.28 & -20 & +10 & 47 & $1.50 \mathrm{~K}$ & $\mathrm{~N}$ & Lap98 \\
\hline $\mathrm{C}^{18} \mathrm{O}(2-1)$ & $219.560 \mathrm{GHz}$ & 8.67 & -20 & +10 & 24 & $3.24 \mathrm{~K}$ & $\mathrm{~N}$ & ZHM00 \\
\hline $\mathrm{CO}(3-2)$ & $345.796 \mathrm{GHz}$ & 11.55 & -20 & +10 & 20 & $47.4 \mathrm{~K}$ & $\mathrm{~N}$ & Lap98 \\
\hline $\mathrm{CS}(2-1)$ & $97.981 \mathrm{GHz}$ & $9.76 / 10.42$ & -40 & +30 & 50 & $4.80 / 0.74 \mathrm{~K}$ & $\mathrm{Y}$ & ZMT95 \\
\hline $\mathrm{CS}(2-1)$ & $97.981 \mathrm{GHz}$ & 9.3 & 0 & 0 & 50 & $3.61 \mathrm{~K}$ & $\mathrm{~N}$ & B96 \\
\hline $\mathrm{CS}(2-1)$ & $97.981 \mathrm{GHz}$ & 9.90 & -40 & +30 & 51 & $5.34 \mathrm{~K}$ & $\mathrm{Y}$ & Lap98 \\
\hline$C^{34} S(2-1)$ & $96.413 \mathrm{GHz}$ & 9.66 & -40 & +30 & 50 & $0.55 \mathrm{~K}$ & $\mathrm{~N}$ & ZMT95 \\
\hline$C^{34} S(2-1)$ & $96.413 \mathrm{GHz}$ & 9.27 & -20 & +10 & 52 & $0.80 \mathrm{~K}$ & $\mathrm{~N}$ & Lap98 \\
\hline $\mathrm{CS}(3-2)$ & $146.969 \mathrm{GHz}$ & 10.42 & -20 & +10 & 34 & $8.92 \mathrm{~K}$ & $\mathrm{Y}$ & Lap98 \\
\hline $\mathrm{CS}(5-4)$ & $244.936 \mathrm{GHz}$ & 9.47 & -20 & +10 & 22 & $7.19 \mathrm{~K}$ & $\mathrm{~N}$ & Lap98 \\
\hline $\mathrm{CS}(7-6)$ & $342.883 \mathrm{GHz}$ & 9.38 & -10 & +10 & 20 & $9.83 \mathrm{~K}$ & $\mathrm{~N}$ & Lap98 \\
\hline $\mathrm{CH}_{3} \mathrm{OH}$ & $6.669 \mathrm{GHz}$ & 4 & -40 & +40 & $2 \times 10^{2}$ & $1.2 \mathrm{Jy}$ & $\mathrm{N}$ & C95 \\
\hline $\mathrm{CH}_{3} \mathrm{OH}$ & $6.669 \mathrm{GHz}$ & 4 & - & - & $2 \times 10^{2}$ & $1.2 \mathrm{Jy}$ & $\mathrm{N}$ & W97 \\
\hline $\mathrm{CH}_{3} \mathrm{OH}$ & $95.169 \mathrm{GHz}$ & $9.3 / 9.8$ & -15 & +15 & 52 & 7.0/21.0 Jy & $\mathrm{N}$ & Val00 \\
\hline $\mathrm{H}_{2} \mathrm{O}$ & $22.235 \mathrm{GHz}$ & 9 & 0 & -10 & $2.4 \times 10^{2}$ & $9.0 \times 10^{2} \mathrm{Jy}$ & $\mathrm{N}$ & SB80 \\
\hline HNCO & $219.798 \mathrm{GHz}$ & 9.92 & -30 & +10 & 24 & $0.24 \mathrm{~K}$ & $\mathrm{Y}$ & ZHM00 \\
\hline Continuum & $5.0 \mathrm{GHz}$ & - & +112 & -51 & $2.6 \times 10^{2}$ & $4.1 \mathrm{Jy}$ & $\mathrm{N}$ & $\mathrm{CH} 87$ \\
\hline Continuum & $1.3 \mathrm{~mm}$ & - & - & - & - & $4.3 \mathrm{Jy}$ & $\mathrm{N}$ & Li92 \\
\hline Continuum & $6.67 \mathrm{GHz}$ & - & +88 & +113 & 1 & $<13 \mathrm{mJy}$ & $\mathrm{N}$ & W98 \\
\hline Continuum & $8.64 \mathrm{GHz}$ & - & +88 & +113 & 1 & $14 \mathrm{mJy}$ & $\mathrm{N}$ & W98 \\
\hline $\mathrm{H} 109 \alpha$ & $5.009 \mathrm{GHz}$ & 1 & +112 & -51 & $2.6 \times 10^{2}$ & $0.083 \mathrm{~K}$ & $\mathrm{~N}$ & CH87 \\
\hline $\mathrm{H} \alpha$ & $6563 \AA$ & - & - & - & - & - & $\mathrm{N}$ & RCW \\
\hline
\end{tabular}

summarized. Numerous molecular transitions have been detected, with critical densities $\left(n_{\mathrm{c}}\right)$ for excitation in a wide range, from $n_{\mathrm{c}}\left[\mathrm{C}^{18} \mathrm{O}\right]=1.5 \times 10^{3} \mathrm{~cm}^{-3}$ up to $n_{\mathrm{c}}[\mathrm{CS}(2-1)]=5.9 \times$ $10^{5} \mathrm{~cm}^{-3}$ and $n_{\mathrm{c}}[\mathrm{CS}(3-2)]=1.4 \times 10^{6} \mathrm{~cm}^{-3}$, which confirms the existence of high-density regions in the molecular cloud associated with IRAS 09149-4743.

The maps obtained in the CS and HNCO lines (Zinchenko et al. 2000; Lapinov et al. 1998) revealed a high-density region located approximately $30-50$ arcsec NW of the central IRAS position (Fig. 1). However, it was the continuum emission detected at 5, 6.67, and $8.64 \mathrm{GHz}$ (Caswell \& Haynes 1987; Walsh et al. 1998) that established this cloud as a region of young massive stars. The discovery of methanol emission at $6.67 \mathrm{GHz}$, generally found very near $\mathrm{O}$ or early-B type stars, led to the conclusion that there is an ultracompact (UC) HII region associated with the IRAS source (Caswell et al. 1995; Walsh et al. 1997). Unfortunately, as Table 1 shows, the spatial resolution of all these observations is too poor to identify the precise location of the ionizing sources in the cloud and most differences in position among the various detections are smaller than the $H P B W$ of the observations. The association of infrared sources with newborn stars is also difficult, because the IRAS ellipse is too large to distinguish small features even in nearby clouds. Persson \& Campbell (1987) detected a near-infrared counterpart of the IRAS source at $16^{\prime \prime} \mathrm{NW}$ of the central IRAS position but inside the error ellipse. Its $J-H$ and $H-K$ colour indices correspond to $A_{\mathrm{V}} \simeq 10 \mathrm{mag}$, and indicate that the star has some excess at $\lambda=2.2 \mu \mathrm{m}$. More recently, two mid-infrared sources were found in the MSX survey (Egan \& Price 1996): one at the position of the IRAS source and a second one associated with a small subcluster of near-infrared stars. The completion of the 2MASS survey allowed a more precise identification of the nearinfrared counterparts of this young stellar cluster. In the next section our near-infrared observations of this cluster are described.

\section{The near-infrared observations}

The near-infrared observations were carried out at "Pico dos Dias" Observatory, southeast Brazil, which is operated by the National Laboratory for Astrophysics (LNA). The near-infrared camera used for the observations (CamIV, Barbosa 2000) contains a Hawaii $1024 \times 1024$ pixel $\mathrm{HgCdTe}$ array detector and $J, H$, and $K$ filters $^{1} . J$ and $H$ filters have a broad response whereas $K$ is centred at $2.138 \mu \mathrm{m}$ and is just $0.022 \mu \mathrm{m}$ wide. By choosing a narrow-band $K$ filter centred at this wavelength the continuum can be better determined because one avoids possible Brackett- $\gamma$ emission which has been observed towards other star forming regions (Roman-Lopes \& Abraham 2006a,b). The images in the $J$ and $H$ bands were obtained with CamIV attached to the 60 -cm Boller \& Chivens telescope, which produces $8^{\prime} \times$ $8^{\prime}$ frames and $0.48^{\prime \prime}$ per pixel. $K$ band images were obtained with the 1.6-m Perkin-Elmer telescope, which gives $4^{\prime} \times 4^{\prime}$ frames and $0.24^{\prime \prime} /$ pixel. The images in the $J$ and $H$ bands were obtained from a single, $300 \mathrm{~s}$ exposure, whereas the $K$-band final image resulted of a set of short exposures, which combined amount to $4224 \mathrm{~s}$ of integration. This long integration time in the $K$ band is due to the lack of a Lyot stop, necessary to reduce the thermal noise, which on the other hand allows the use of CamIV in different telescopes. Figure 1 shows the combined $K$-band frame, which is approximately centred at the IRAS source position.

The data were reduced using the IRAF ${ }^{2}$ astronomical package, version 2.12.2, following a standard routine described by Roman-Lopes et al. (2003), Roman-Lopes \& Abraham (2004), and briefly described here. PSF photometry was obtained

\footnotetext{
${ }^{1}$ http://www.Ina.br/opd/instrum/Camiv/filtros.htm

2 IRAF is distributed by the National Optical Astronomy Observatory, which is operated by the Association of Universities for Research in Astronomy, Inc., under contract to the National Science Foundation.
} 


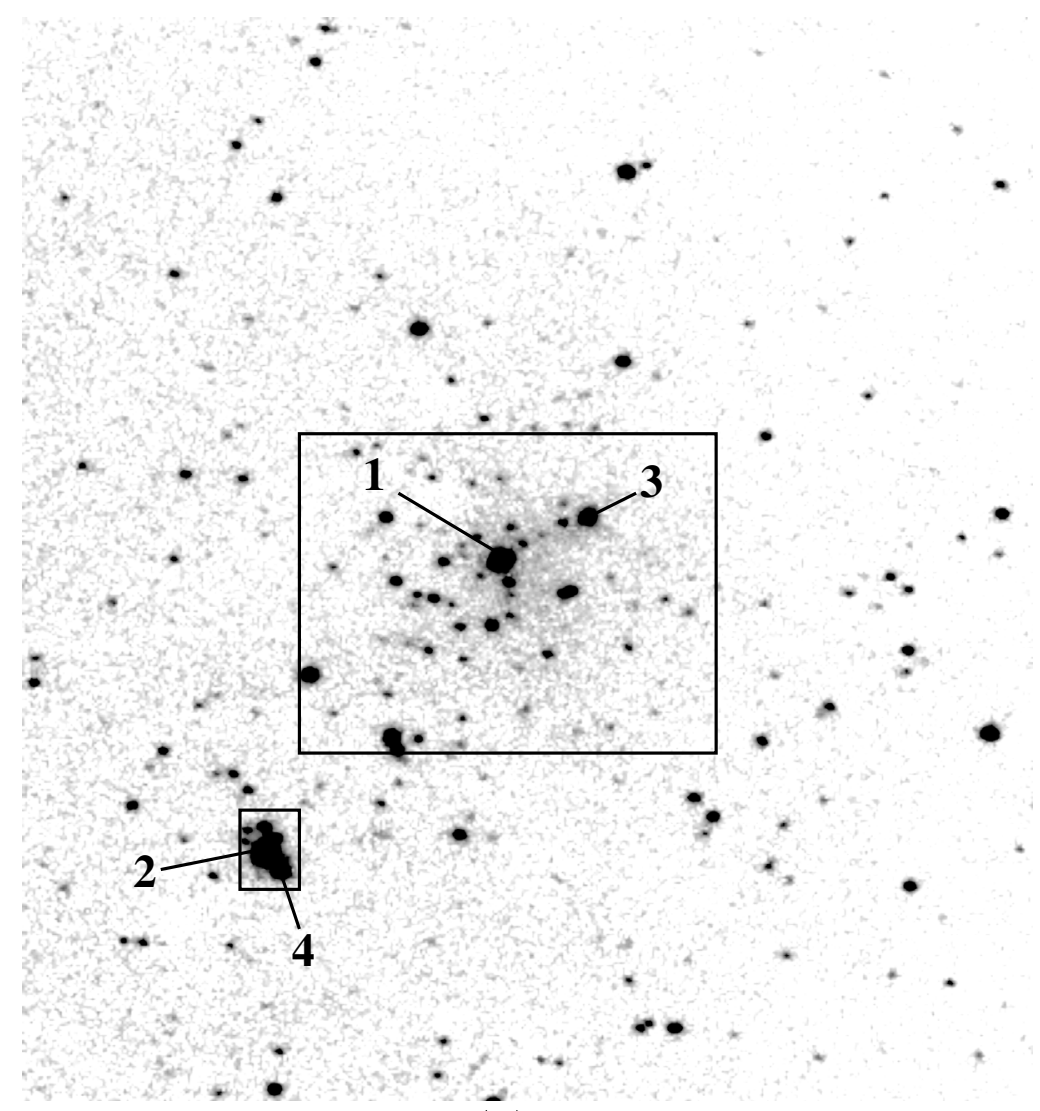

(a)

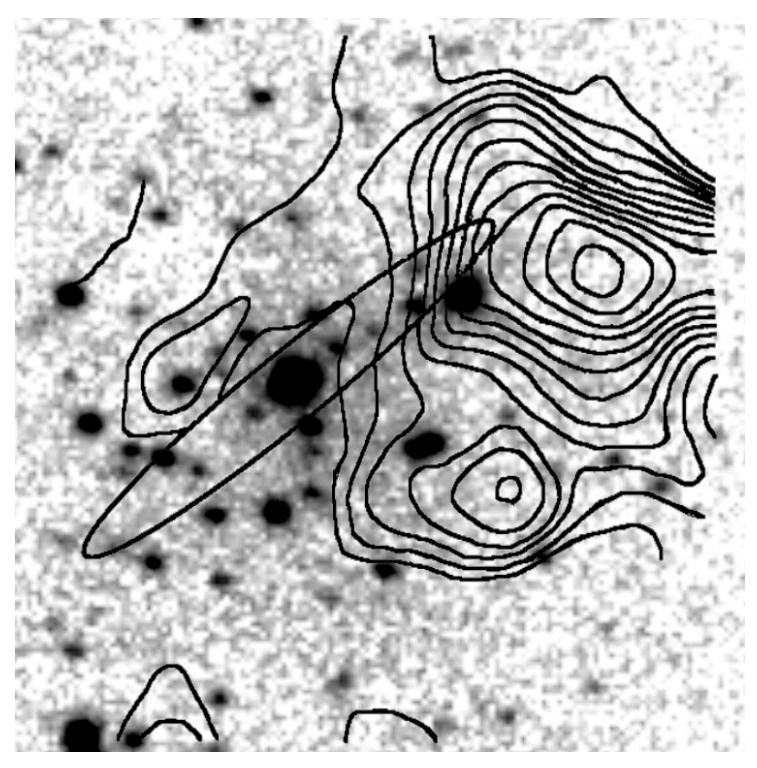

(b)

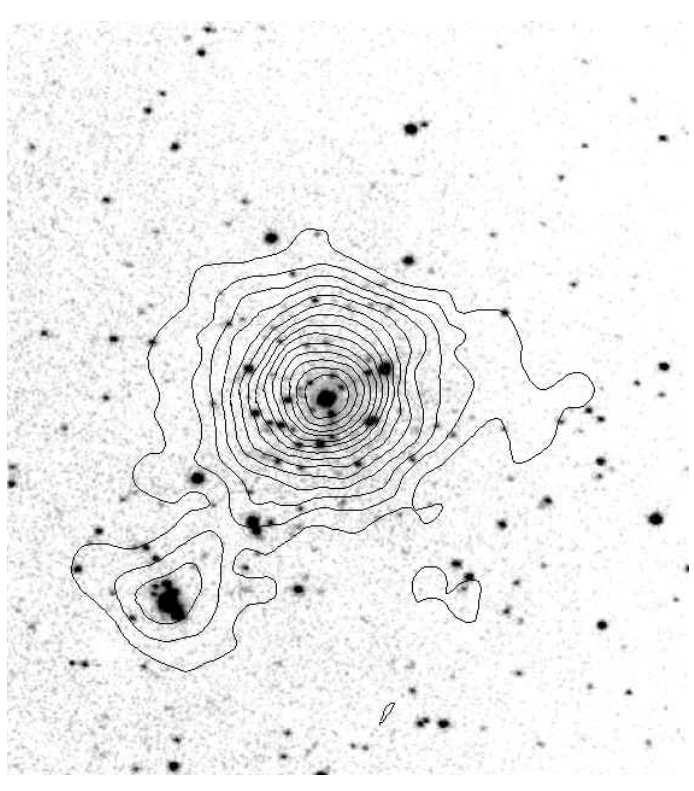

(c)

Fig. 1. a) $K$-band image of the cluster associated with IRAS 09149-4743. The large box delimits the central part of the cluster, as described in the text. The small cluster lies 1.1' SE of the centre (the foot-like structure inside the small box), which contains sources number \#2 (the brightest, at the centre of the group) and \#4 (at the heel of the foot). The frame measures $3.1^{\prime} \times 3.5^{\prime}$ in size; north is to the top and east to the left; b) detailed view of the central part of the cluster, showing the IRAS error ellipse and the HNCO radio map as observed by Zinchenko et al. (2000), superposed to the $K$-band image; c) MSX isophotes at 14.7 micron ( $D$-band), the isophotes start at $I=2.3 \times 10^{-6} \mathrm{~W} \mathrm{~m}^{-2} \mathrm{sr}^{-1}$ with steps of $I=1.0 \times 10^{-6} \mathrm{~W} \mathrm{~m}^{-2} \mathrm{sr}^{-1}$, the $H P B W$ is $18^{\prime \prime}$.

using the PSF algorithm ALLSTAR, which is included in the DAOPHOT package (Stetson 1987). We chose to use PSF instead of aperture photometry because several parts of the cluster look crowded. The PSF radii used were 11 pixels for the $J$ and $H$ bands and 13 pixels for the $K$ band. An examination of the images showed that the average $F W H M$ of the stars during the observations were $2.8,2.9$, and 5.4 pixels for the $J, H$, and $K$ bands, respectively (or $\sim 1.3$ arcsec for the three bands). As a 

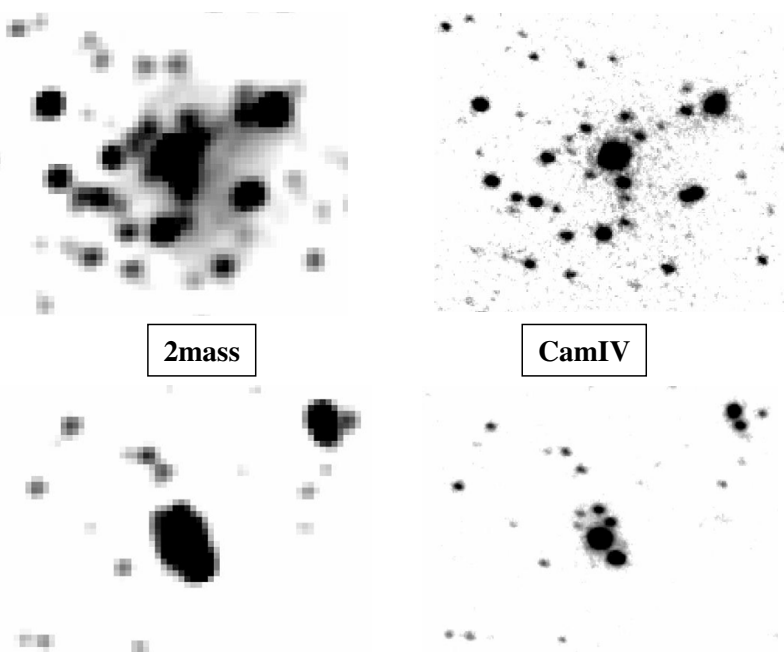

Fig. 2. Comparison between the images obtained during the 2MASS survey (left) and this work (right), in the $K$ band.

comparison, the full-width at half maximum measured on stars of the corresponding 2MASS image produced FWHM $=3^{\prime \prime}$ on average, which is more than twice the figures obtained by CamIV. Figure 2 shows a sample of two images of the cluster, obtained by 2MASS and CamIV, both centred on the core of the cluster. The CamIV images are superior, showing numerous resolved sources which appear blended in the 2MASS images. An example is the small cluster that resembles a "foot"like structure in the lower part of the image; two sources appear blended in the 2MASS image and listed in the point-source catalogue, whereas six stars are clearly visible in the CamIV image.

After reduction, because of the dithering technique used during the observations, the size of the cluster frame was reduced to $5.2^{\prime} \times 4.3^{\prime}$ for the $J$ and $H$ bands, and $3.1^{\prime} \times 3.5^{\prime}$ for the $K$-band. The three frames are centred near the IRAS source position, which corresponds also to the centre of the cluster and their boundaries are the following: $9^{\mathrm{h}} 16^{\mathrm{m}} 27.4^{\mathrm{s}}<\alpha(\mathrm{J} 2000.0)<$ $9^{\mathrm{h}} 16^{\mathrm{m}} 58.7^{\mathrm{s}},-47^{\circ} 58^{\prime} 23^{\prime \prime}<\delta(\mathrm{J} 2000.0)<-47^{\circ} 54^{\prime} 05^{\prime \prime}$ for the $J$ and $H$ bands, and $9^{\mathrm{h}} 16^{\mathrm{m}} 33.6^{\mathrm{s}}<\alpha(\mathrm{J} 2000.0)<9^{\mathrm{h}} 16^{\mathrm{m}} 52.3^{\mathrm{s}}$, $-47^{\circ} 58^{\prime} 07^{\prime \prime}<\delta(\mathrm{J} 2000.0)<-47^{\circ} 54^{\prime} 39^{\prime \prime}$ for the $K$ band.

The zero-point constants for photometry were obtained comparing instrumental "CamIV" and 2MASS magnitudes of a set of stars common to both frames. This set comprised only sources that appear isolated in both the 2MASS and CamIV $J, H$, and $K$ images, covering a wide range of magnitudes and colours. The errors on the zero point magnitudes are estimated as 0.016 , 0.019 , and $0.023 \mathrm{mag}$ for the $J, H$ and $K$ bands respectively. The final photometric errors are calculated as the quadratic sum of the zero-point errors given above and the intrinsic uncertainty of each star, given by the ALLSTAR routine. The signal-to-noise ratio in the CamIV images $S N R=5$ corresponds to the following magnitudes: $J=17.0, H=16.3$, and $K=15.0$.

Figure 3 shows a comparison between CamIV and 2MASS magnitudes of the sets of sources used as calibrators. The correlation is very close to linear, as the result of the appropriate derivation of the point-source function and the inexistence of a colour-dependent term which correlates the CamIV and 2MASS photometric systems (Roman-Lopes \& Abraham 2006b).

Table 2, available at the CDS, contains position and $J H K$ photometry of all sources located within the rectangles drawn in Fig. 1a, which contain sources with high membership probability. Column 1 lists the order number which designates the sources in this work (\#); Cols. 2 and 3 give the J2000.0 equatorial coordinates $(\alpha, \delta)$; Cols. 4 through 9 give the $J, H$, and $K$ magnitudes, with their corresponding errors $\left(J, \sigma_{J}, H, \sigma_{H}\right.$, $\left.K, \sigma_{K}\right)$.

\section{The cluster}

\subsection{Colour-colour distribution}

Because of the presence of the dark molecular cloud containing species such as CS and other radicals typical of high density regions, stellar magnitudes are expected to be severely affected by extinction, whereas other sources may show some infrared excess due to the presence of warm circumstellar dust. Either caused by high extinction or circumstellar infrared emission, some stars belonging to the cluster have high colour indices. In order to compare probable members of the cluster with field stars, a non-crowded "comparison field" among the 2MASS images was chosen for study, situated about 3.6' west of the cluster centre, delimited by coordinates: $9^{\mathrm{h}} 16^{\mathrm{m}} 9.6^{\mathrm{s}}<\alpha(\mathrm{J} 2000)<9^{\mathrm{h}} 16^{\mathrm{m}} 26.4^{\mathrm{s}}$ and $-48^{\circ} 00^{\prime} 00^{\prime \prime}<$ $\delta(\mathrm{J} 2000)<-47^{\circ} 51^{\prime} 00^{\prime \prime}$. Figure 4 shows the location of the comparison field relative to the cluster frame.

The comparison field is not located completely out of the molecular cloud $A$. The CO maps (Dame et al. 1987; Murphy \& May 1991) reveal that cloud $A$ of VMR is about one degree in radius, which includes the region of the comparison field. However, as the observations show that the $\mathrm{CO}$ column density decreases monotonically with the distance from the central part of the cluster, the extinction in the direction of the comparison field is expected to be much lower. Figure 5 confirms this hypothesis: the "cluster" frame contains sources showing $H-K$ up to 2.0 whereas the colour indices of the comparison frame are limited to $H-K<1.0$.

Figure 6 shows $(H-K) \times(J-H)$ colour-colour diagrams of the "comparison" and "cluster" frames. A comparison between the distribution of the $J-H$ index of the two frames reveals that this colour cannot be used as a discriminator between cluster and field stars, since the $J-H$ distribution in both frames is similar. On the other hand, the distribution of the $H-K$ index, shown in Fig. 5, can possibly be an indicator of the presence of a stellar cluster, either in a star forming phase or in an evolved state, deeply embedded in a dark cloud. In Fig. 6a the majority of the sources showing $H-K$ excess have $J-H$ as a lower limit, as the arrows indicate. Therefore it is possible that at least a fraction of these objects do not have anomalous infrared excesses at all, and the extinction towards them may be so high as to make their fluxes in the $J$ band undetected. Figure 5, which shows the distribution of the $H-K$ colour index irrespective of the detection in the $J$ band, confirms that the frame that contains the cluster is more reddened than the comparison frame. However, a significant fraction of the members of the cluster exhibit low values of $H-K$. For instance, sources \#1, \#2, and \#4 in Figs. 1 and 6 all have $H-K<1.0$, even though they have a high probability of membership, as the image of the cluster shows.

Figure $6 \mathrm{a}$ also shows that the real members are found not only near the central crowd, but are spread over the whole cluster frame. Sources situated in the central region of the cluster and in the small "foot"-like cluster seen in Fig. 1 have a high membership probability, and are represented as filled circles. These two areas correspond to the following limits, identified as two boxes in Fig. 1: $9^{\mathrm{h}} 16^{\mathrm{m}} 40.0^{\mathrm{s}}<$ $\alpha(J 2000)<9^{\mathrm{h}} 16^{\mathrm{m}} 47.0^{\mathrm{s}},-47^{\circ} 57^{\prime} 00^{\prime \prime}<\delta(\mathrm{J} 2000)<-47^{\circ} 56^{\prime} 00^{\prime \prime}$ 

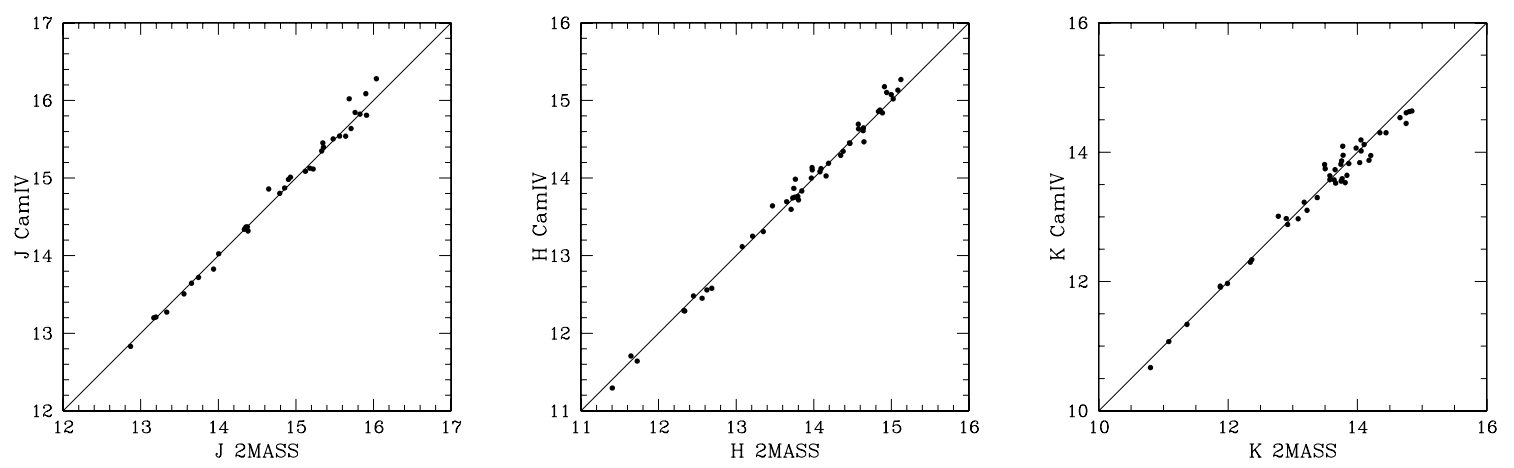

Fig. 3. CamIV and 2MASS magnitudes for the calibration stars. The straight line denotes a one-to-one correspondence between the two systems.

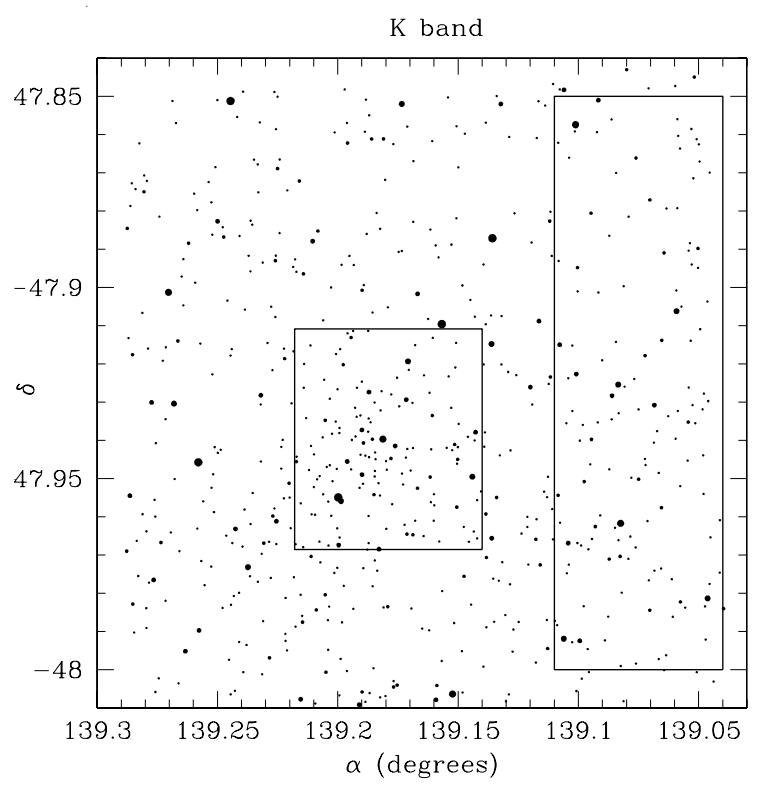

Fig. 4. Positions of the "cluster" field (box in the centre), which corresponds to the area in the sky observed with CamIV, and the "comparison" field (the rectangle on the right side). Boundaries are defined in Sect. 4, given in J2000.0 coordinates. Infrared sources appear as filled circles with diameters proportional to their $K$ magnitude.

(central cluster), and $9^{\mathrm{h}} 16^{\mathrm{m}} 47.3^{\mathrm{s}}<\alpha(J 2000)<9^{\mathrm{h}} 16^{\mathrm{m}} 48.6^{\mathrm{s}}$, $-47^{\circ} 57^{\prime} 25^{\prime \prime}<\delta(J 2000)<-47^{\circ} 57^{\prime} 10^{\prime \prime}$ ("foot"-like cluster). All objects located within these two coordinate boxes and detected either in the $J H K$ or $H K$ bands are listed in Table 2. When comparing sources in and outside the boxes, there is no significant difference between the distributions of their colour indices. A possible interpretation is that the extinction is spread over the whole region surveyed, as the maps of molecular emission (Dame et al. 1987; Murphy \& May 1991; Zinchenko et al. 2000) indicate.

As seen in Table 2, source \#1 is the brightest object in the central part of the cluster and it lies at the centre of the IRAS error ellipse. Alone, it accounts for the majority of the near-infrared flux within the ellipse. Besides the photometry presented in this paper, Liseau et al. (1992) obtained JHKL magnitudes of the central source (IRS 49/1) using an aperture photometer. Their measurements differ by less than 0.15 mag from the present photometry in the $J, H$ and $K$ bands. The 2MASS magnitudes of this source also differ by less than $0.10 \mathrm{mag}$ from the present work. Altogether, these agreements indicate that, although the central part of the cluster appears crowded, especially in the 2MASS images (Fig. 2), the

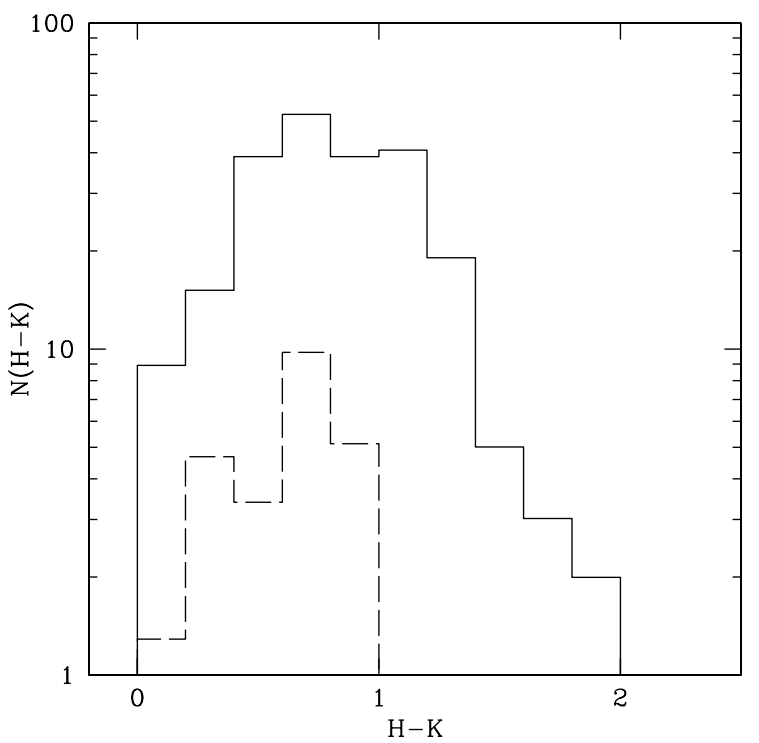

Fig. 5. Histograms of the sources detected in the $H$ and $K$ bands, and showing $K<14.0$, which corresponds to the completeness limit of the survey. Continuous and dashed lines correspond to sources in the cluster and comparison frames, respectively. All figures have been normalized to correspond to the same area in the sky.

contribution of the neighbouring objects to the flux of source \#1 is negligible.

Figure 7 shows the spectral energy distribution (SED) of source \#1, including the MSX source G270.2596+00.8351, the counterpart of IRAS 09149-4743 situated inside the IRAS error ellipse and at the centre of the isophotes drawn in Fig. 1c. The SED does not correspond to a class I (Wilking et al. 1989) source, as had been found by Liseau et al. (1992). Except for the A-band photometry (at $8.3 \mu \mathrm{m}$ ), the MSX flux densities are lower than the IRAS measurements at similar wavelengths. This discrepancy may be caused by the beam width of the "Spirit" camera used in the MSX survey, which is smaller than IRAS, and that would collect less radiation coming from neighbouring stars and/or warm dust.

Source \#2 is the brightest among the six stars that comprise the small cluster in the small box shown in Fig. 1 and it is brighter than the central source itself. This object is clearly resolved in the CamIV images, although it appears saturated in the $J$ and $H$ images. Because of this, we preferred to use the 2MASS magnitudes for this source instead of CamIV's. As seen in the lower part of Fig. 2, the 2MASS survey does not resolve the six stars which comprise that small cluster. The sources identified in Table 2 as \#31, \#32, \#43 and \#50, together with 


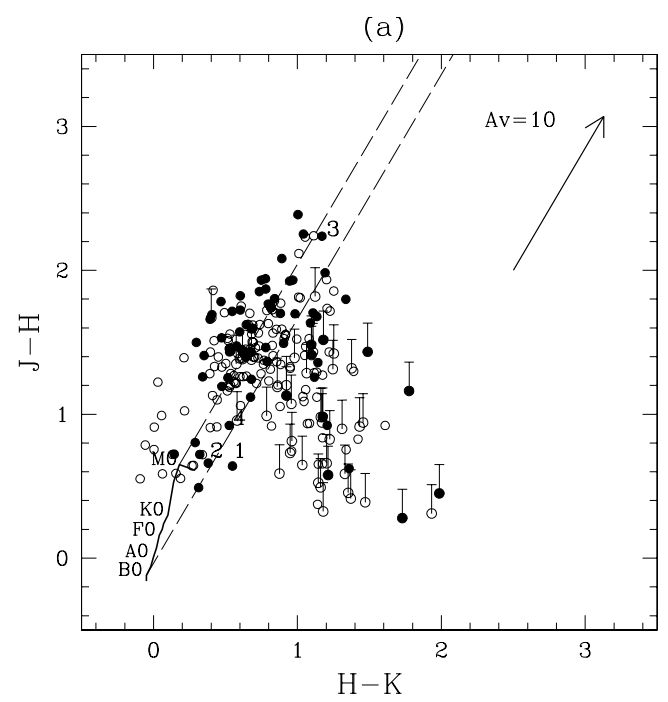

(b)

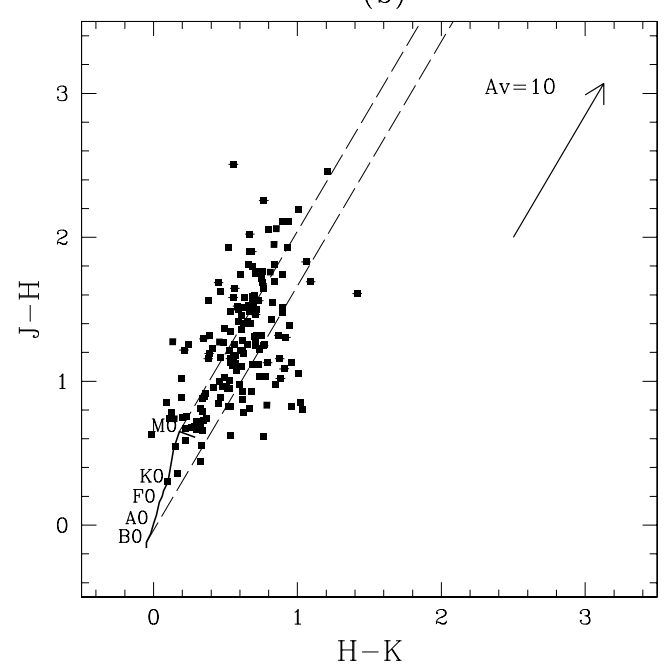

Fig. 6. a) Colour-colour diagram of the cluster. Sources drawn as filled circles are located within the boxes shown in Fig. 1a, whereas the remaining objects in the frame are open circles. Upward bars designate sources not detected in the $J$ band. The arrow represents the reddening vector for $A_{V}=10 \mathrm{mag}$, and the dashed lines delimit the region of reddened main-sequence. Some sources identified by numbers ( 1 to 4 ) correspond to the objects numbered in Fig. 1 and Table 2. b) Colour-colour diagram of the comparison field, delimited by the box shown in Fig. 4.

source \#2 appear as a single object in the 2MASS images and catalogue. However the contribution of these four sources to the flux of source \#2 is of little significance: if excluded, its magnitudes would be increased by $0.07,0.09$, and $0.10 \mathrm{mag}$ in the $J, H$, and $K$ bands, respectively. We conclude that these stars do not significantly affect the classification of source \#2, based on its 2MASS magnitudes. In the JHK colour-colour diagram, source \#2 appears on the ribbon that represents the normally reddened main sequence, and similarly to source \#1, its visual extinction is less than $10 \mathrm{mag}$. The MSX counterpart to this source is G270.2795+00.8353, but most of its mid-infrared flux is probably associated with the whole compact "foot"-like cluster shown in Fig. 1, which is smaller than the spatial resolution of MSX. Unlike source \#1, two objects may contribute more significantly to the near-infrared flux of this compact cluster: sources \#2 and \#4, and it is possible that they comprise the major part of the mid-infrared flux as well. The MSX image in

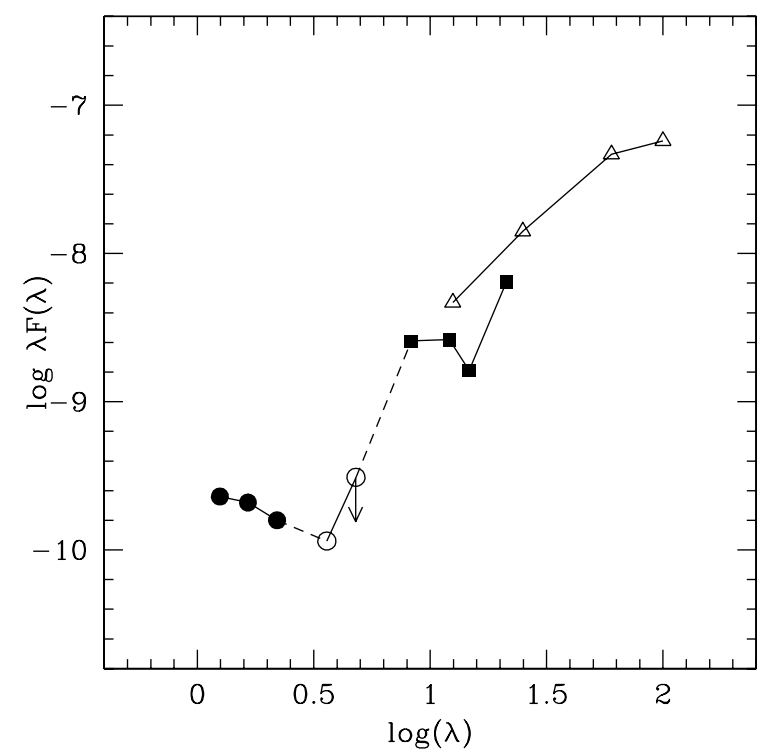

Fig. 7. The spectral energy distribution of the central source of the cluster. The wavelength ( $x$-axis) is given in micron, and $\lambda F(\lambda)$ in $\mathrm{erg} \mathrm{s}^{-1} \mathrm{~cm}^{-2}$. The data refer to: this work (filled circles), Liseau et al. 1992 (open circle), MSX point-source catalogue (squares), and IRAS (triangles).

the D band shown in Fig. 1c suggests that the infrared emission at $\lambda=14.7 \mu \mathrm{m}$ follows the general spatial distribution of stars.

\subsection{The colour-magnitude diagram}

As discussed in the introduction, the stellar cluster is associated with an HII region. Therefore, some of its most luminous members must be stars of early-B spectral type at least, to supply a significant number of ionizing photons. Irrespective of the distance to the cluster, the $\mathrm{C}-\mathrm{M}$ diagram given in Fig. 8 can be used to determine dereddened $J$ magnitudes, which can be associated with stellar luminosities and masses if the distance to the cluster is known. According to this method, dereddened $J$ magnitudes are obtained by applying the dereddening vector to the points in the C-M diagram up to the point where they intercept the ZAMS curve. Two objects are more likely to be the ionizing sources of the HII region: stars \#1 and \#2. Star \#4 is far less luminous, whereas little can be concluded about source \#3, which is one of the redder objects in the cluster as Fig. 6 shows: the extinction towards it exceeds 20 mag. In fact, this star appears in a region of high molecular column density, as the HNCO isophotes in Fig. 1b reveal. This star might also be a background late-type giant, which appears red in the colour-colour diagram due to its low colour temperature associated with severe reddening.

Cloud $A$, which belongs to the VMR and is seen towards the cluster, had its distance determined by Murphy \& May (1991), who used the CO line to derive its kinematic distance as $D_{\text {kin }}=1 \mathrm{kpc}$. Assuming that the cluster and the molecular cloud are situated at the same distance and that the stars are in the ZAMS phase, the object identified as \#1 is an early-B star. The HNCO map shown in Fig. 1 reveals a decrease in molecular density around this source, which suggests that the star has swept out the molecular gas in its neighbourhood. This observation confirms that this star is closely related to the molecular cloud and that its high-energy photons have already begun the process of destroying the grains and molecules in the central part 


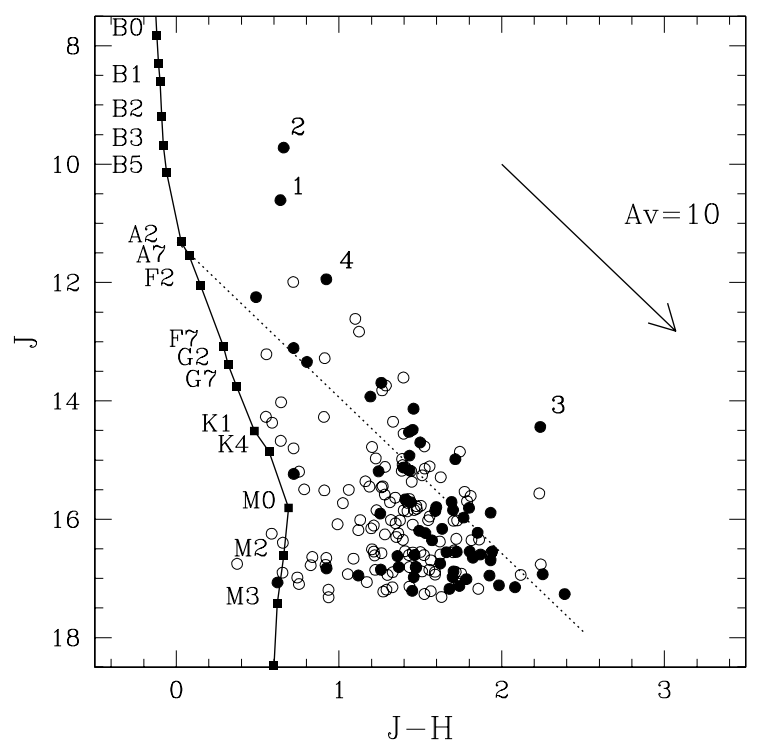

Fig. 8. Colour-magnitude diagrams of the stellar cluster associated with IRAS 09149-4743. The dotted line delimits the boundary above which the diagram is statistically complete. Similarly to Fig. 6a, sources in the central part of the cluster and in the tiny cluster are represented as filled circles whereas open circles represent the remaining objects of the frame. A general interstellar extinction law is assumed, which corresponds to $E(J-H) / E(H-K)=1.50$, (van der Hulst 1946; Becklin et al. 1978) or $A_{\mathrm{J}} / E(J-H)=2.79$, and $D=1.0 \mathrm{kpc}$ (Murphy \& May 1991).

of the cluster. Star \#2, situated at the small "sub-cluster", seems to be more luminous than \#1, and is probably the main ionizing source of the HII region. Spectroscopy in the near-infrared would help to determine their spectral types more accurately, whereas high-resolution radio observations would help to reveal the distribution of the ionized gas among these stars.

Both the colour-colour and the colour-magnitude diagrams show that the average visual extinction of the cluster is about $10 \mathrm{mag}$. The diagonal dotted line that crosses the colour-magnitude diagrams from bottom right to the upper left corner delimits the region where the survey is statistically complete concerning its mass distribution. According to these diagrams, and assuming the distance to the cluster as $1 \mathrm{kpc}$, the survey is statistically complete down to spectral type A5 at worse, which corresponds to $m \simeq 2 M_{\odot}$. There are forty stars above this limit and a significant number of low-mass stars as well: between ten and thirty early M-type stars have been detected in the survey.

\section{Conclusions}

An infrared survey of the cluster associated with IRAS 09149-4743 was carried out in the $J, H$, and $K$ bands with a spatial resolution superior to that of the 2MASS survey. 62 stars situated in the central part of the cluster, that look unresolved in the 2MASS images were distinguished and their magnitudes determined. The main visual extinction of the stars is about $10 \mathrm{mag}$, but it can be over $20 \mathrm{mag}$ in some cases. Two stars appear as candidates to ionize the
HII region: one located at the centre of the cluster, associated with IRAS 09149-4743, and a second one, located $1.1^{\prime}$ to the southeast, which seems to be the main ionizing source of the region. There is also a third source that can possibly play an important role in the ionization process, however it is too reddened to allow an accurate determination of its spectral type. Additional near and mid-infrared observations (such as those provided by "Spitzer"), including spectroscopy, would help to clarify the real nature of these objects.

Acknowledgements. The authors thank the anonymous referee for valuable suggestions and a critical reading of the manuscript. This research has made use of the SIMBAD database, operated at CDS, Strasbourg, France. This work was supported by FAPESP (Fundação de Amparo à Pesquisa do Estado de São Paulo, Brazil), and CNPq (Conselho Nacional de Desenvolvimento Científico e Tecnológico, Brazil) in the context of the "Millennium Institute" entitled Evolução de estrelas e galáxias na era dos grandes telescópios. We acknowledge the support provided by the LNA staff during the observations.

\section{References}

Barbosa, C. L. D. R. 2000, Master's Dissertation, Divisão de Astrofisica, INPE/MCT, 81p.

Becklin, E. E., Matthews, K., Neugebauer, G., \& Willner, S. P. 1978, ApJ, 220, 831

Brand, J., Blitz, L., \& Wouterloot, J. G. A. 1986, A\&AS, 65, 537

Bronfman, L., Nyman, L.-A., \& May, J. 1996, A\&A, 115, 81 (B96)

Caswell, J. L., \& Haynes, R. F. 1987, A\&A, 171, 261 (CH87)

Caswell, J. L., Vaile, R. A., Ellingsen, S. P., Whiteoak, J. B., \& Norris, R. P. 1995, MNRAS, 272, 96 (C95)

Dame, T. M., Ungerechts, H., Cohen, R. S., et al. 1987, ApJ, 322, 706

Dutra, C. M., Bica, E., Soares, J., \& Barbuy, B. 2003, A\&A, 400, 533

Egan, M. P., \& Price, S. D. 1996, AJ, 112, 2862

Lapinov, A. V., Schilke, P., Juvela, M., \& Zinchenko, I. I. 1998, A\&A, 336, 1007 (Lap98)

Liseau, R., Lorenzetti, D., Nisini, B., Spinoglio, L., \& Moneti, A. 1992, A\&A, 265, 577 (Li92)

Massi, F., Giannini, T., Lorenzetti, D., et al. 1999, A\&AS, 136, 471

Massi, F., Lorenzetti, D., Giannini, T., \& Vitali, F. 2000, A\&A, 353, 598

Massi, F., Lorenzetti, D., \& Giannini, T. 2003, A\&A, 399, 147

May, J., Murphy, D. C., \& Thaddeus, P. 1988, A\&AS, 73, 51

Menten, K. M. 1991, ApJ, 380, L75

Murphy, D. C., \& May, J. 1991, A\&A, 247, 202 (MM91)

Persson, S. E., \& Campbell, B. 1987, AJ, 94, 416

Rodgers, A. W., Campbell, C. T., \& Whiteoak, J. B. 1960, MNRAS, 121, 103 (RCW)

Roman-Lopes, A. 2004, Ph.D. Thesis, Departamento de Astronomia, IAG/USP

Roman-Lopes, A., \& Abraham, Z. 2004, AJ, 127, 2817

Roman-Lopes, A., \& Abraham, Z. 2006a, AJ, 131, 951

Roman-Lopes, A., \& Abraham, Z. 2006b, AJ, 131, 2223

Roman-Lopes, A., Abraham, Z., \& Lépine, J. R. D. 2003, AJ, 126, 1896

Scalise, E., \& Braz, M. A. 1980, A\&A, 85, 149 (SB80)

Skrutskie, et al. 1997, in The Impact of Large Scale Near-IR Sky Surveys, ed. F. Garzon et al. (Netherlands: Kluwer), 25

Snell, R. L., Mundy, L. G., Goldsmith, P. F., Evans II, N. J., \& Erickson, N. R. 1984, ApJ, 276, 625

Stetson, P. B. 1987, PASP, 99, 191

Val'tts, I. E., Ellingsen, S. P., Slysh, V. I., et al. 2000, MNRAS, 317, 315 (Val00)

van der Hulst, H. C. 1946, Recherches Astron. Obs. Utrecht, 11, part 1

Walsh, A. J., Hyland, A. R., Robinson, G., \& Burton, M. G. 1997, MNRAS, 291, 261 (W97)

Walsh, A. J., Burton, M. G., Hyland, A. R., \& Robinson, G. 1998, MNRAS, 301, 640 (W98)

Wilking, B. A., Lada, C. J., \& Young, E. T. 1989, ApJ, 340, 823

Wouterloot, J. G. A., \& Brand, J. 1989, A\&AS, 80, 149 (WB89)

Zinchenko, I., Mattila, K., \& Toriseva, M. 1995, A\&AS, 111, 95 (ZMT95)

Zinchenko, I., Henkel, C., \& Mao, R. Q. 2000, A\&A, 361, 1079 (ZHM00) 